using tartrate-resistant acid phosphatase (TRAP) staining and bone resorption assay, respectively. Osteoblast differentiation was assessed by alkaline phosphatase staining and activity was determined by calcium measurement in the supernatant.

Results Trabecular bone volume, thickness and number as well as cortical volume and thickness, and femur length were significantly lower in 12-week-old IL-23R $\mathrm{R}^{-/-}$mice compared to WT. In addition, three-point bending data revealed reduced maximum force in IL-23R $\mathrm{R}^{-/}$femurs. Surprisingly, bone volume was similar between both groups at the age of 26 weeks. However, similar to 12 -week-old mice, endocortical volume and femur perimeter were significantly lower in $\mathrm{IL}-23 \mathrm{R}^{-/-}$mice compared to WT. To further study the temporal differences in bone phenotype, we studied osteoclasts and osteoblasts from 7- and 12-week-old mice in vitro. Osteoclast differentiation and function were similar at both ages between WT and IL$23 \mathrm{R}^{-/-}$mice. Interestingly, $\mathrm{BM}$ cells of 7 -week-old IL-23R $\mathrm{R}^{-/-}$ mice had reduced capacity to differentiate towards osteoblasts, compared to WT. In contrast, these cells showed higher differentiation and significantly higher calcium uptake than WT mice at 12 weeks.

Conclusions IL-23R ${ }^{-/-}$mice have temporally dynamic changes in bone metabolism, which is possibly related to alterations in osteoblasts, however, the exact mechanism still needs to be elucidated.

Disclosure of Interest None declared.

\section{P121/021 MIR-146A AN IMPORTANT KEY PLAYER IN BONE METABOLISM}

${ }^{1} V$ Saferding*, ${ }^{1} \mathrm{M}$ Hofmann, ${ }^{1} \mathrm{JS}$ Brunner, ${ }^{1} \mathrm{MF}$ Militaru, ${ }^{1} \mathrm{~A}$ Puchner, ${ }^{1} \mathrm{~S}$ Hayer, ${ }^{2} \mathrm{M}$ Timmen, ${ }^{2} \mathrm{R}$ Stange, ${ }^{1} \mathrm{JS}$ Smolen, ${ }^{1} \mathrm{~S}$ Blüml. ${ }^{1}$ Medical University of Vienna, Vienna, Austria; ${ }^{2}$ University Hospital Muenster, Muenster, Germany

\subsection{6/annrheumdis-2018-EWRR2019.109}

Career situation of first and presenting author Post-doctoral fellow.

Introduction Micro RNAs (miRNAs) play a crucial role in the regulation of bone metabolism. MiR-146a, an important antiinflammatory miRNA, was found to negatively impact osteogenesis and bone regeneration in vitro, by controlling the differentiation of mesenchymal stem cells. But to date the role of miR-146a in bone remodelling, its influence on bone stability and development of osteoporosis is not known.

Objectives Our aim is to analyse the function of miR-146a in bone metabolism.

Methods Systemic bone, tibiae and femur, of wt and miR146a deficient animals was assessed histologically and via $\mu \mathrm{CT}$ analysis, over a period of 3 to 18 months of age. Serum cytokine levels were analysed by Elisa. MRNA expression levels in bone were analysed by qPCR. To induce osteoporosis, ovariectomy (OVX) induced bone loss was performed.

Results When we analysed bone volume of long bones histologically as well as with $\mu \mathrm{CT}$ analysis we detected significantly increased trabecular bone mass in miR-146a deficient compared to wt animals, starting at an age of 6 months. In addition cortical thickness of systemic bones from miR-146a knock out animals was significantly increased compared to control mice. Analysis of serum in aged miR-146a deficient animals displayed elevated activity of bone resorbing osteoclasts as amounts of CTX I in miR-146 $\mathrm{a}^{-/-}$mice were significantly increased compared to wt animals. Q-PCR analysis of important osteoclast as well as osteoblast marker genes in bones ex vivo displayed elevated expression of signature molecules of both cell types in aged miR-146a deficient mice, suggesting a regulatory role of miR-146a in both osteoclasts as well as osteoblasts. When we induced osteoporosis using the OVX disease model, histological analysis of long bones showed significant trabecular bone loss in ovariectomized wt mice. In contrast, we detected no trabecular bone loss in ovariectomized miR-146a knock out animals, suggesting that loss of miR-146a deficiency protects bone loss induced by estrogen deficiency.

Conclusions MiR-146a seems to control bone turnover and miR-146a deficient mice accrue bone over time. Moreover this miRNA has a negative influence on bone loss occurring during oestrogen loss induced osteoporosis. Therefore miR146 a could be possibly used as a therapeutic target in the treatment of osteoporosis.

Disclosure of Interest None declared.

\section{P122 IMPORTANT ROLE OF DENDRITIC CELLS IN INFLAMMATORY ARTHRITIS}

${ }^{1} E$ Simader*, ${ }^{1} \mathrm{~A}$ Puchner, ${ }^{1} \mathrm{~V}$ Saferding, ${ }^{1} \mathrm{E}$ Goncalves-Alves, ${ }^{2} \mathrm{R}$ Pfeifle, ${ }^{3} \mathrm{G}$ Krönke, 'I Smolen, 'S Blüml. 'Rheumatology, Medical University of Vienna, Vienna, Austria; ${ }^{2}$ Rheumatology, Universitätsklinikum Erlangen; ${ }^{3}$ Rheumatology, Universitätsklinikum Erlangen, Erlangen, Germany

\subsection{6/annrheumdis-2018-EWRR2019.110}

Career situation of first and presenting author Student for a master or a $\mathrm{PhD}$.

Introduction Important role of dendritic cells in inflammatory arthritis.

Objectives Investigation of the role of $\mathrm{CD} 11 \mathrm{c}^{+}$cells in joint inflammation and destruction.

Methods We analyzed histological sections of $\mathrm{K} / \mathrm{BxN}$ serum transfer arthritis as well as hTNFtg arthritis for the presence of $\mathrm{CD}_{11 \mathrm{c}^{+}}$cells by immunohistochemistry. We used CD11cdiphteria toxin receptor (DTR) transgenic mice. $\mathrm{K} / \mathrm{BxN}$ serum transfer arthritis was induced, and mice were given either DT or PBS or in wt and BARF3 deficient mice. In addition CD11c DTR mice were crossed into hTNFtg animals and also received either DT or PBS. The severity of arthritis was determined clinically and histologically.

Results Both $\mathrm{CD}^{+} \mathrm{CD} 11 \mathrm{c}^{+}$and $\mathrm{CD} 11 \mathrm{~b}^{+} \mathrm{CD} 11 \mathrm{c}^{+}$, can be found in synovial tissue in TNF driven arthritis. Upon depletion of $\mathrm{CD}_{11 \mathrm{c}^{+}}$cells clinical signs of $\mathrm{K} / \mathrm{BxN}$ serum transfer arthritis were significantly reduced. Histological analysis found reduced synovial inflammation after the depletion of $\mathrm{CD} 11 \mathrm{c}^{+}$ cells in $\mathrm{K} / \mathrm{BxN}$ arthritis. In addition, local bone destruction and the number of osteoclasts was also significantly reduced. In addition to $\mathrm{K} / \mathrm{BxN}$ arthritis, we found that also in TNFdriven arthritis depletion of $\mathrm{CD} 11 \mathrm{c}^{+}$cells led to a striking reduction of synovial inflammation and a complete depletion of osteoclasts.

Conclusions These data show that in addition to initiating an adaptive immune response, $\mathrm{CD} 11 \mathrm{c}^{+}$dendritic cells, are also involved in innate effector mechanisms of inflammatory 
arthritis. Especially $\mathrm{CD} 11 \mathrm{~b}^{+} \mathrm{CD} 11 \mathrm{c}^{+}$and monocyte derived inflammatory seem to play a role in inflammatory arthritis, suggesting that they could be an important therapeutic target.

\section{REFERENCES}

1. Sarkar S, Fox DA. Dendritic cells in rheumatoid arthritis. Front Biosci. 2005;10:656-65.

2. Merad M, Sathe P, Helft J, Miller J, Mortha A. The dendritic cell lineage: ontogeny and function of dendritic cells and their subsets in the steady state and the inflamed setting. Annu Rev Immunol 31:563-604.

3. Banchereau J, Steinman RM. Dendritic cells and the control of immunity. Nature 1998;392(6673):245-52.

Acknowledgements We would like to thank the Medical University of Vienna and especially Prof. Smolen for their support and all members of the University of Erlangen for their assistance.

Disclosure of Interest None declared.

\section{P123 EXTRACELLULAR MATRIX ATTENUATED MATRIX- DEGRADING EFFECTS OF VISFATIN DURING ADIPOGENIC MSC DIFFERENTIATION}

${ }^{1} \mathrm{~L}$ Tsiklauri ${ }^{*},{ }^{2} \mathrm{~J}$ Werner, ${ }^{1} \mathrm{~K}$ Frommer, ${ }^{3} \mathrm{~S}$ Rehart, ${ }^{2} \mathrm{~S}$ Wenisch, ${ }^{1} \mathrm{U}$ Müller-Ladner, ${ }^{1}$ E Neumann. 'Department of Internal Medicine and Rheumatology, Kerckhoff-Klinik, Bad Nauheim, Germany, Justus-Liebig-University Giessen, Bad Nauheim; ${ }^{2}$ Institute of VeterinaryAnatomy, -Histology and -Embryology, Clinic of Small Animals, Justus-Liebig-University Giessen, Giessen; ${ }^{3}$ Department of Orthopedics and Trauma Surgery, Markus Hospital, Frankfurt, Germany

\subsection{6/annrheumdis-2018-EWRR2019.111}

Career situation of first and presenting author Student for a master or a $\mathrm{PhD}$.

Introduction Osteoporosis (OP) is the most common agerelated disorder characterized by bone loss and correlates with increased bone marrow adiposity due to a shift of osteogenic towards adipogenic differentiation of bone marrow mesenchymal stem cells (MSC). Therefore, bone marrow adipocytes are in direct contact with the altered bone matrix in OP. Adipose tissue is metabolically active. Therefore, adipocyte-derived factors such as adipokines might influence MSC differentiation.

Objectives We analyzed the presence of adipokines (visfatin, resistin and leptin) in bone tissue and their effects on MSC differentiation under standard culture vs. spongiosa.

Methods RNA and MSC were isolated from spongiosa of femoral heads of osteoarthritis patients after hip replacement surgery, or after osteoporotic femoral neck fracture. Adipogenic MSC differentiation was performed with/without adipokines and visfatin inhibitor Apo866 as well as SB203580 p38MAPK inhibitor. For the transfer and differentiation of MSC on cancellous bone, bone fragments were purified and sterilized. Gene expression was evaluated by Realtime PCR. Protein production was analyzed by ELISA.

Results Visfatin and leptin levels were increased in OP bone vs. non-osteoporotic OA bone, however, resistin was reduced. Visfatin induced the secretion of proinflammatory factors during adipogenesis in standard cell culture as well as on cancellous bone. Visfatin-induced cytokine release was markedly reduced during differentiation on spongiosa (e.g.

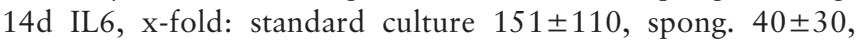
$\mathrm{n}=7$ ). Significantly elevated MMP13 mRNA as well as protein expression induced by visfatin could be observed during adipogenesis in standard cell culture as well as on spongiosa, however visfatin-mediated MMP13 expression was reduced on cancellous bone (e.g. $21 \mathrm{~d}$, x-fold,: standard culture $81 \pm 89$, spong. $13 \pm 21, \mathrm{n}=7$ ). The visfatin inhibitor Apo866 inhibited the visfatin-induced cytokine release. However, the MMP13 expression was not influenced during adipogenesis in culture $(n=4)$. In contrast to Apo866, the p38-MAPK inhibitor did not reduce cytokine release during adipogenesis.

Conclusions Visfatin and leptin levels were elevated in osteoporotic bone. Therefore, visfatin-mediated increase of MMPs and proinflammatory cytokines during adipogenic differentiation might influence bone turnover at the adipose tissue/bone interface. Our results support the idea that the extracellular matrix attenuates visfatin-mediated detrimental effects during adipogenesis. The observed visfatin-mediated effects most likely depend on different signaling pathways.

Disclosure of Interest None declared.

\section{P124/027 OSTEOCYTE-DERIVED PODOPLANIN IS AN IMPORTANT REGULATOR OF BONE REMODELLING IN THE KBXN SERUM TRANSFER MODEL OF RHEUMATOID ARTHRITIS}

${ }^{1} \mathrm{C}$ Wehmeyer*, ${ }^{2} \mathrm{AJ}$ Naylor, ${ }^{3} \mathrm{~K}$ Moeller, ${ }^{4} \mathrm{G}$ Poologasundarampillai, ${ }^{1} \mathrm{~T}$ Pap, ${ }^{2} \mathrm{CD}$ Buckley. ${ }^{1}$ Institute of Musculoskeletal Medicine, University of Muenster, Muenster, Germany; ${ }^{2}$ Institute of Inflammation and Ageing, University of Birmingham, Birmingham, UK; ${ }^{3}$ Faculty of Engineering and Computer Science, University of Applied Science, Osnabrueck, Germany;

${ }^{4}$ School of Dentistry, University of Birmingham, Birmingham, UK

10.1136/annrheumdis-2018-EWRR2019.112

Career situation of first and presenting author Post-doctoral fellow.

Introduction Osteocytes derive from bone-forming osteoblasts and are located deep inside the bone matrix. They are encysted in cavities (lacunae) and form dendritic extensions to develop a dense sentinel network inside the bone. Osteocytes are not passive cells. They modulate bone remodelling through regulation of both osteoclast and osteoblast activity. Immature osteocytes express the transmembrane glycoprotein podoplanin (PDPN/gp38) ${ }^{1}$ which is important for dendrite elongation and osteocyte function. ${ }^{2}$ Moreover it has been reported that PDPN expression is regulated by inflammatory cytokines including TNF $\alpha$, IL- 6 , IL-22, TGF- $\beta 1$, IFN- $\gamma^{3}$ and is highly expressed in synovial tissues from RA patients. ${ }^{4}$ However the role of PDPN in osteocytes during inflammatory disease has not previously been investigated.

Objectives In this study we investigated the effect of osteocyte-specific deletion of PDPN in the $\mathrm{KBxN}$ serum transfer (ST) mouse model of rheumatoid arthritis.

Methods Dmp1 Cre mice were crossed with PDPN ${ }^{\text {flox/flox }}$ mice to generate osteocyte specific conditional knockout mice as well as appropriate $\mathrm{PDPN}^{\text {flox/fox }}$ controls. KBxN ST arthritis was used to study the effect of PDPN deletion on mouse arthritis progression. PDPN expression was assessed by immunohistochemistry. Osteoclast numbers were calculated by TRAP staining. Loss of cartilage and pannus formation was evaluated by Safranin-O and $\mathrm{H}$ and $\mathrm{E}$ staining. Standard microCT and synchrotron microCT analysis was used to assess bone density parameters, osteocyte location and bone erosions.

Results PDPN is expressed in osteocytes at sites of bone erosions in inflamed joints of $\mathrm{KBxN}$ ST mouse model. Osteocytespecific PDPN deletion has no effect on trabecular and cortical bone density parameters in mouse tibiae under resting 